\title{
Enhancing brains
}

Dr Pete Moore 


\section{Societal}
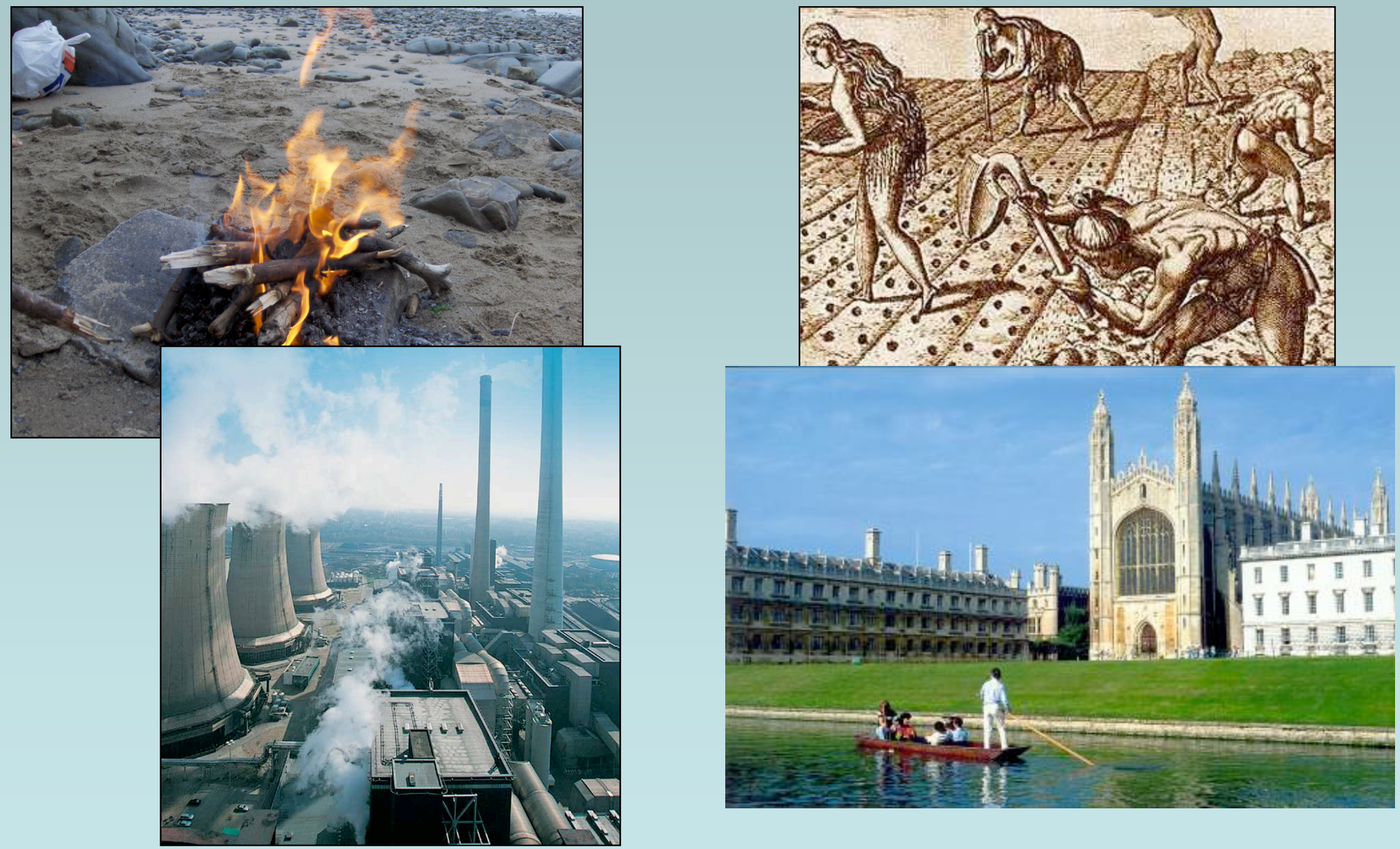

Definitions Cogito... Implants

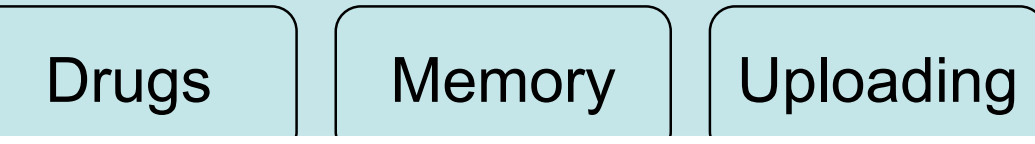




\section{Personal}
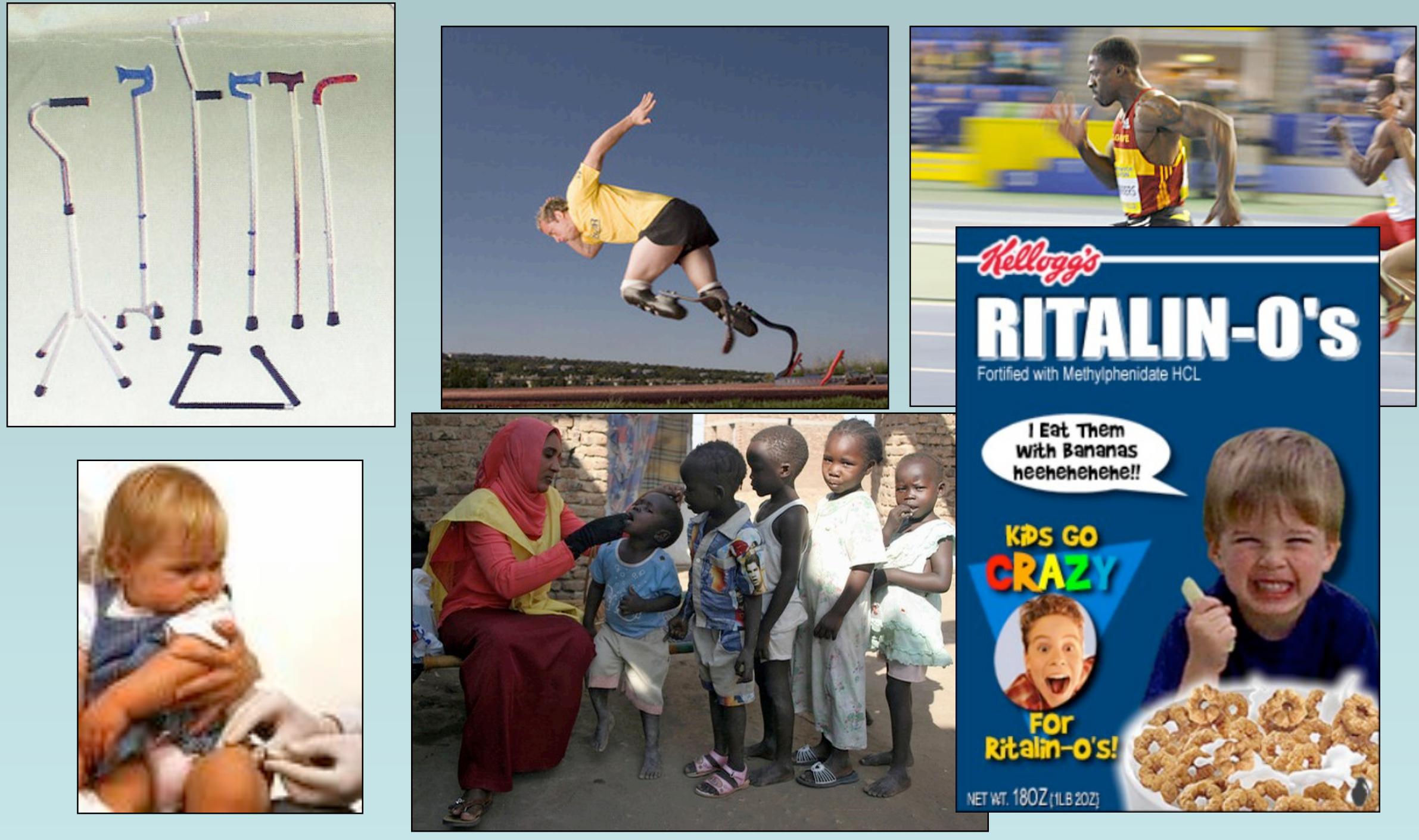

Definitions Cogito... Implants

Drugs

Memory

Uploading 


\section{Species enhancement}
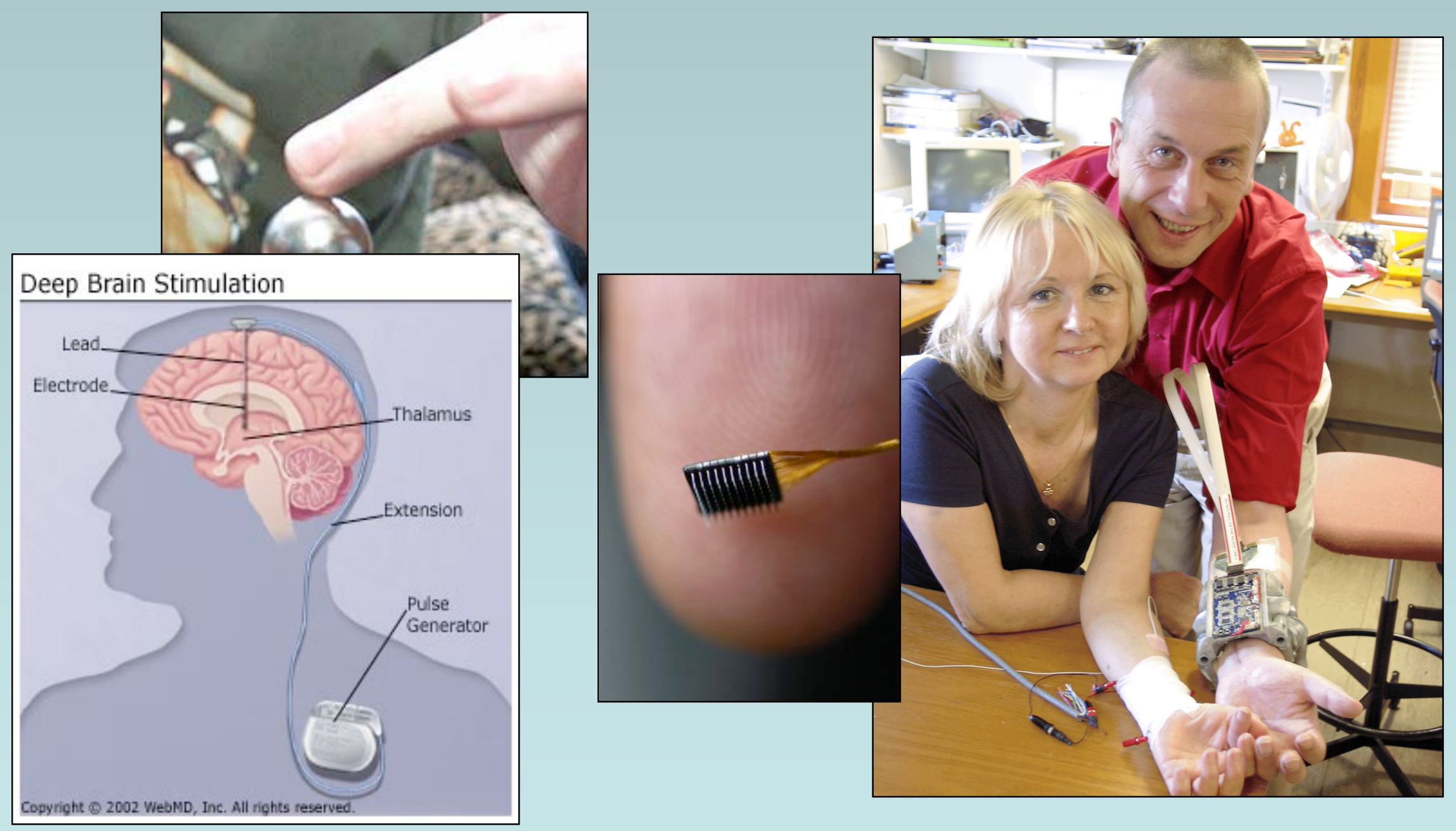

Definitions

Cogito... Implants

Drugs

Memory

Uploading 


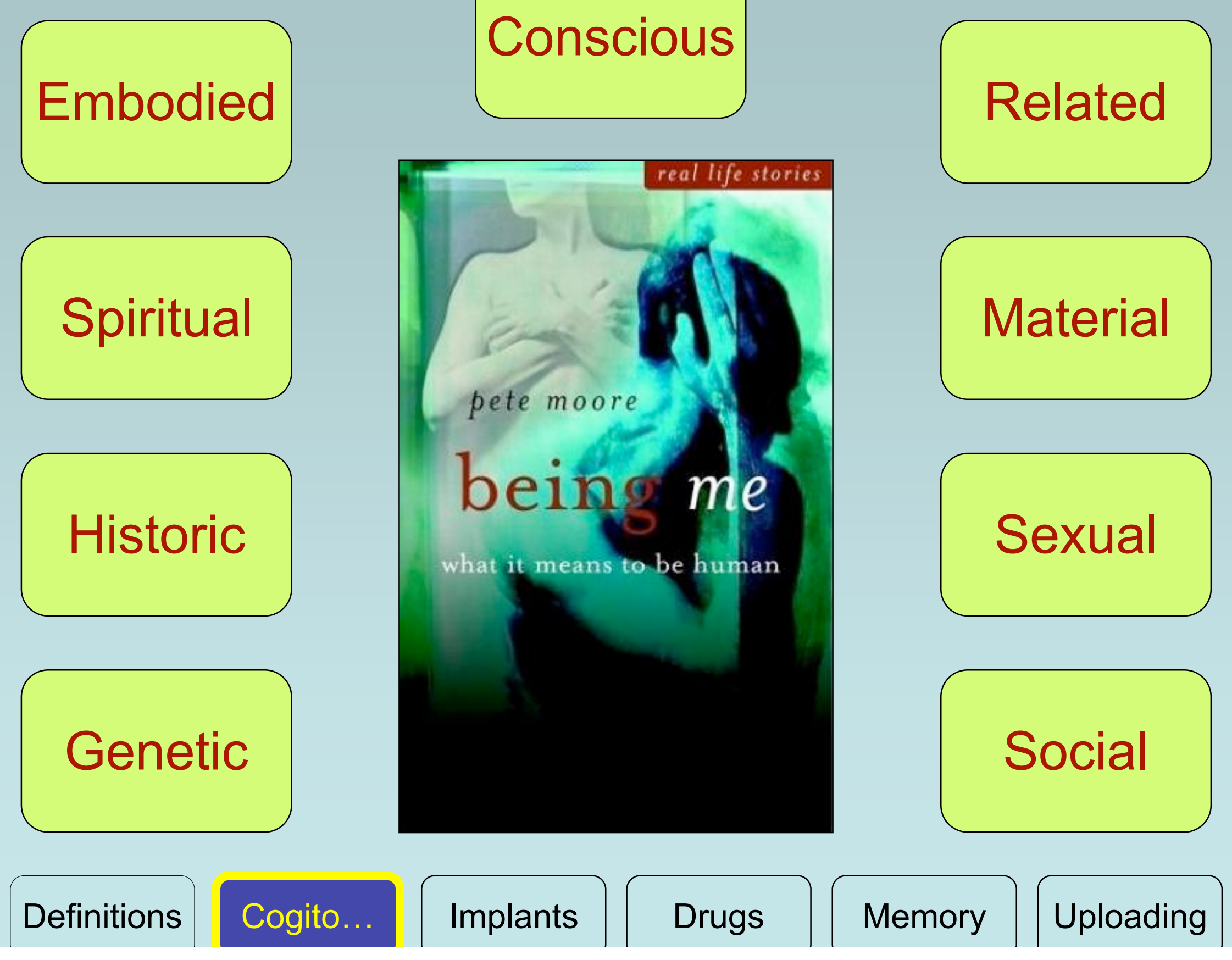




\section{Consciousness}

- What is it?

- How measure it?

- direct electrical / actions / MRI / PET scans

\begin{tabular}{|c|c|c|c|c|}
\hline Definitions & Cogito... & Implants & Drugs & Memory \\
\hline
\end{tabular}




\section{Historic development}

- blood

- heart

- brain

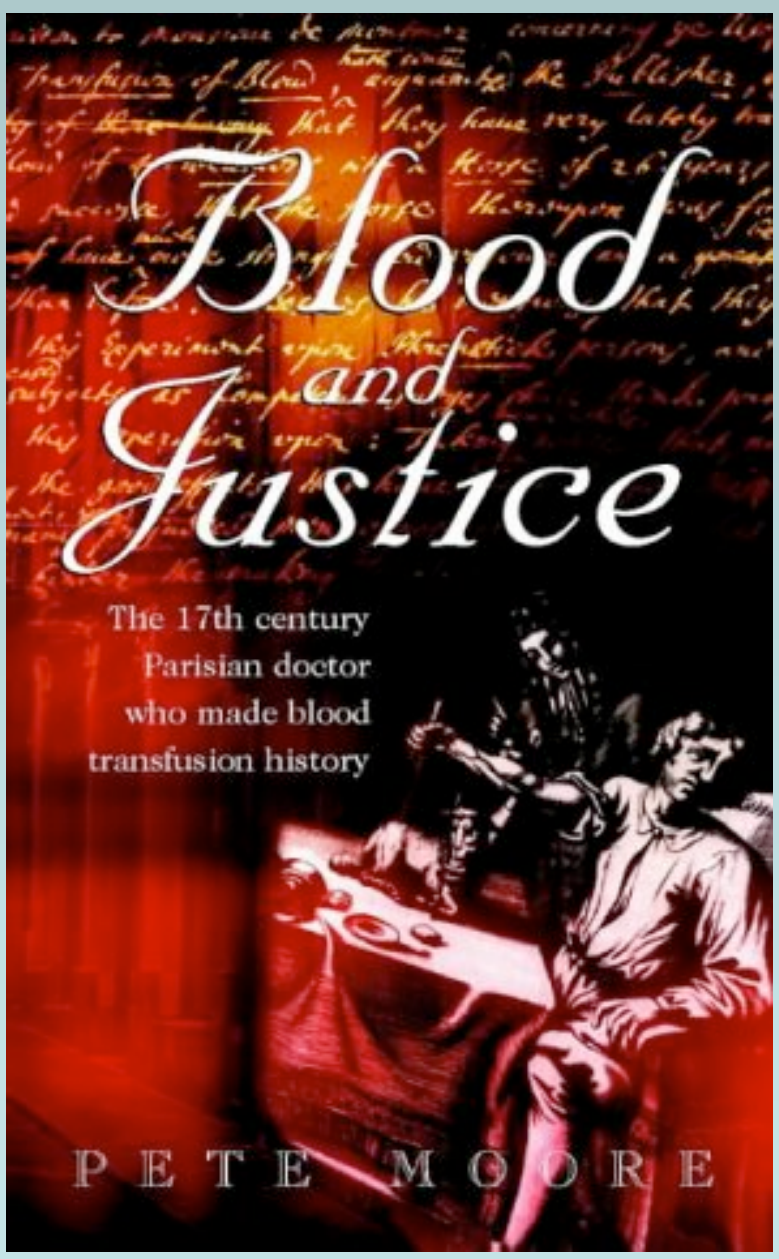

Definitions

Cogito...

Implants

Drugs

Memory

Uploading 


\section{Enhancing plug-ins}

- Cochlear

- Eye

- Deep Brain Stimulation

?+ve

?-ve

\section{Deep Brain Stimulation}

$\nabla$

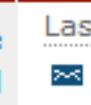

$\infty$

Electrode

By
Sci

Thalamus

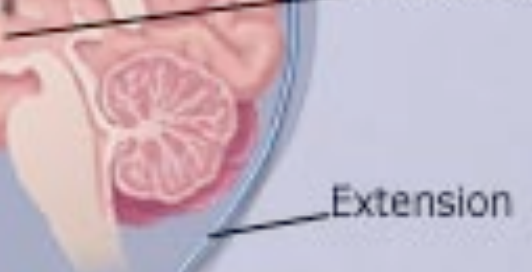

in Co

el pa

"W Copyright 2002 WebMD, Inc. All rights reserved.

Money Life \& style

$$
\text { calmera aाru curver? }
$$

would iu unn-ctart the ntherwice hline eve and allnw natientc

al sight

t

gain

Generator
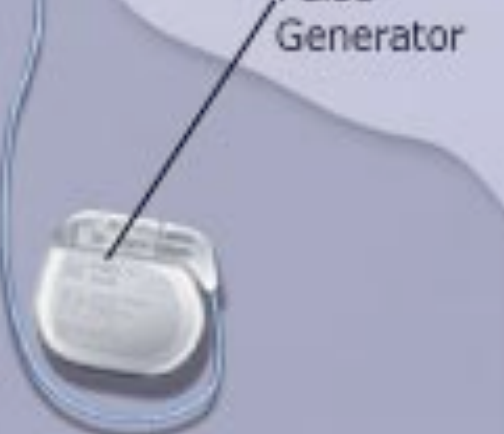

stores only very

ssful that its

histicated version

Definitions Cogito... 


\section{Every day boosters}

- Alcohol

- Caffeine

- Nicotine
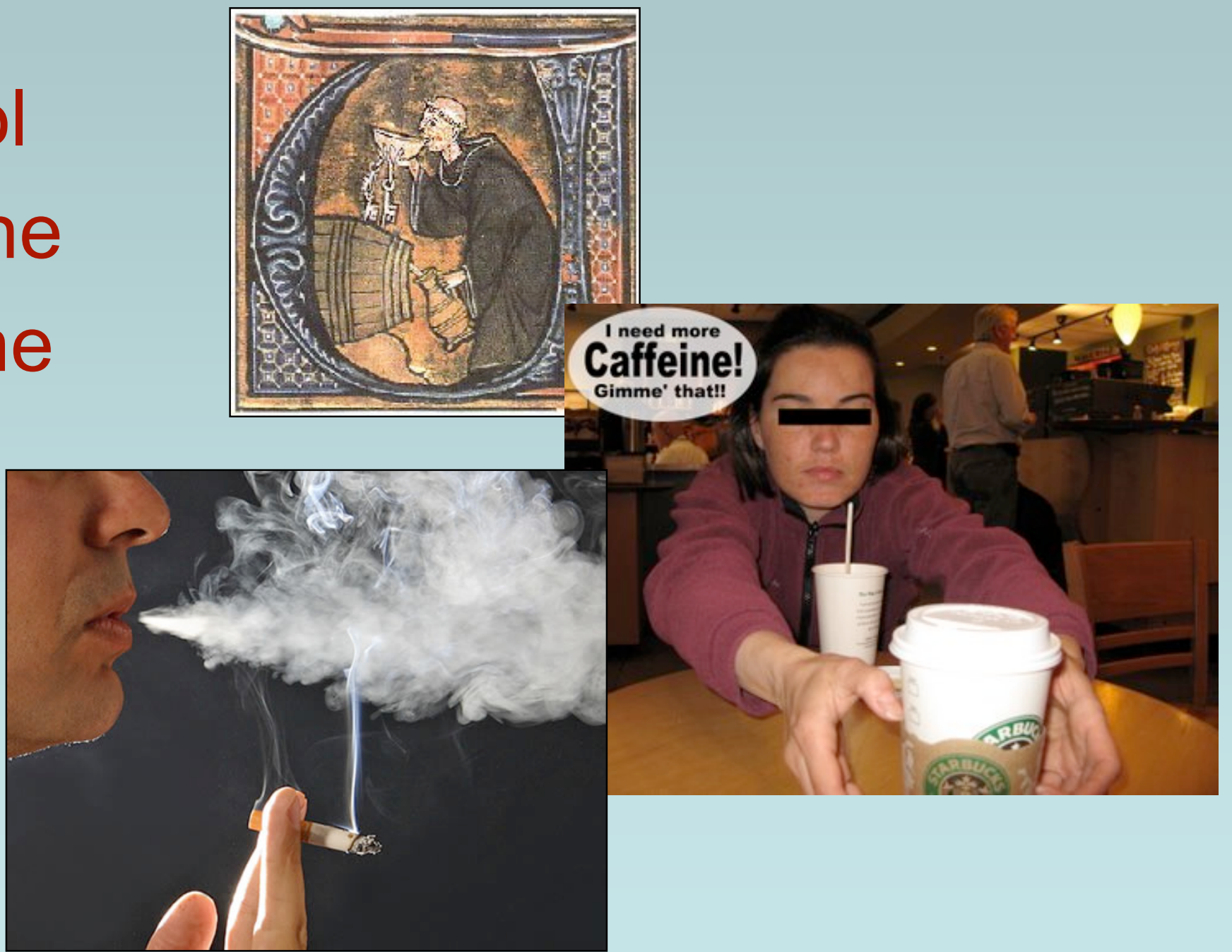

Definitions Cogito... Implants 


\section{Joshua Foe}

- Ritalin / Adderall

- First hour - mildly euphoric

- Then calmly focussed

- But "intellectually blinkered"

- Memory training

- game of golf

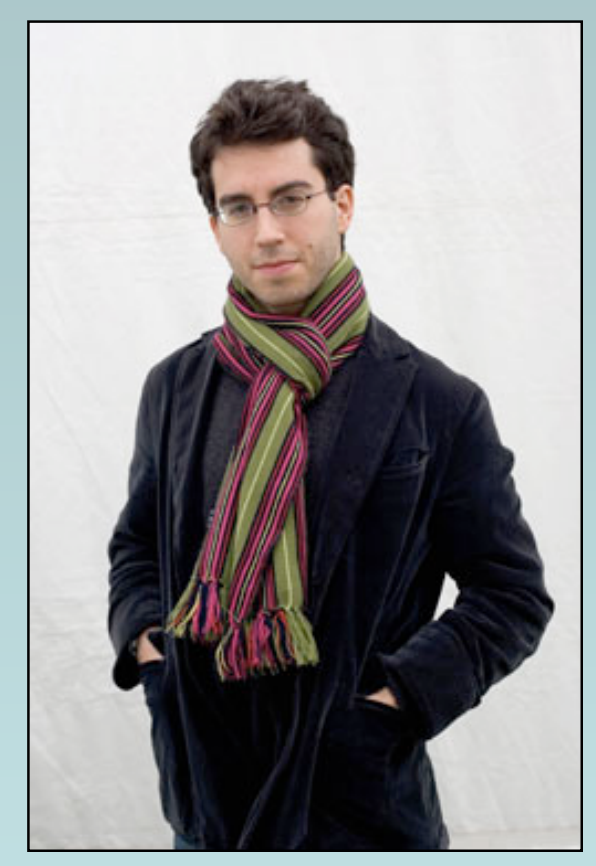

- US national champion - pack of cards in 1'40" world record $=0$ '30"

$\begin{array}{llll}\text { Definitions } & \text { Cogito... } & \text { Implants } & \text { Drugs } \\ \text { Memory } & \text { Uploading }\end{array}$




\section{Enhanced concentration}

- 17 April 2002

- Canadian Light Infantry Battle Group Kandahar

- Training event

- Hit by 'friendly fire' from F16

- Dextroamphetamine

- banned after first gulf war - reintroduced

- focussed - awake - BUT - paranoid

$$
\text { Cogito... Implants }
$$




\section{Enhanced memory}

- What would it look like?

\section{- Remember more}

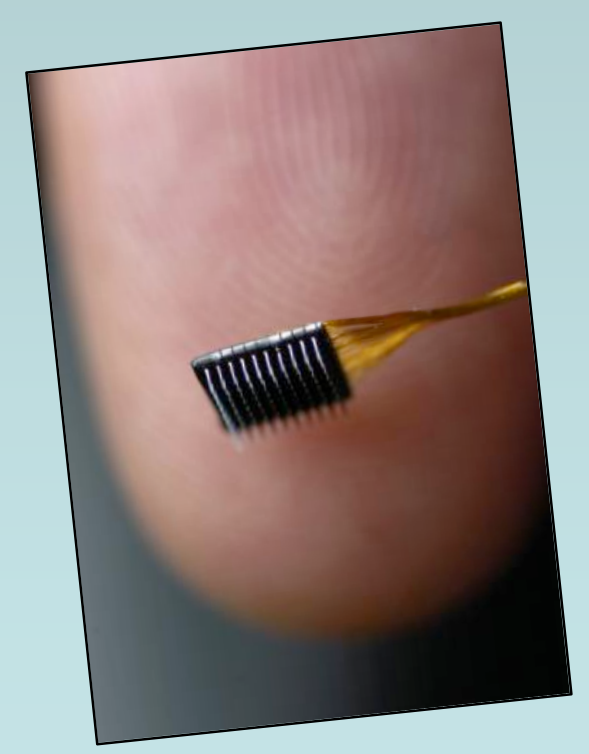

\section{guardian.co.uk}

Search:

News $\mid$ Sport $\mid$ Comment $\mid$ Culture $\mid$ Business $\mid$ Money $\mid$ Life \& style $\mid$ Travel $\mid$ Environment Blos

\section{News > World news}

Brain implant may restore memory

Tim Radford, science editor

The Guardian, Thursday March 13, 2003

Article history

\section{国回曰国}

US researchers are poised to test an artificial aid to the brain. They have made a mathematical model of a memory store called the hippocampus, and programmed it on to a silicon chip. This could one day be fitted to a

human with brain damage from stroke, epilepsy or dementia.

Futurologists have talked for more than a decade about "downloading"

human memory on to a chip and preserving it as an implant. But for the

first time, some scientists think they can see a way to do it.

The hippocampus is one of the most studied parts of the brain. It

processes experiences so that they can be stored as memories. Last

A larger|smaller

World news

Education

Higher education

Research - Science

Society

Health

Life and style

Health \& wellbeing

UK news 


\section{Enhanced memory}

- What would it look like?

- Remember more

- File more efficiently

\begin{tabular}{|l|l|l|l} 
Definitions & Cogito... & Implants & Drugs \\
Uploading
\end{tabular}




\section{Enhanced memory}

- What would it look like?

- Remember more

- File more efficiently

- Remember less

\begin{tabular}{|c|c|c|}
\hline \multicolumn{2}{|c|}{ 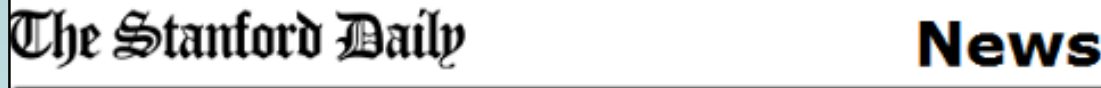 } & \\
\hline \multicolumn{2}{|c|}{ THURSDAY April 15, 2004} & Search \\
\hline Home & \multirow{4}{*}{$\begin{array}{l}\text { What if you could erase your memory? } \\
\text { April 15, } 2004 \\
\text { By Abigail MCDaniel }\end{array}$} & \\
\hline OTHER ISSUES & & Share Tools \\
\hline Archives & & Email \\
\hline & & $\begin{array}{l}\text { Print } \\
+ \text { +Share }\end{array}$ \\
\hline THIS Issue & \multirow{2}{*}{\multicolumn{2}{|c|}{$\begin{array}{l}\text { Imagine that at the age of } 15 \text { you were violently sexually assaulted. Ever since, } \\
\text { you have been unable to enter into a close relationship, as any intimate contact } \\
\text { provokes strings of vivid nightmares and flashbacks during which you remember } \\
\text { each moment of the attack in excruciating detail. At some point, you would }\end{array}$}} \\
\hline $\begin{array}{l}\text { Front page } \\
\text { News } \\
\text { Sports } \\
\text { Opinions } \\
\text { Intormicsion }\end{array}$ & & \\
\hline
\end{tabular}

Definitions

\section{Cogito... Implants}

Drugs

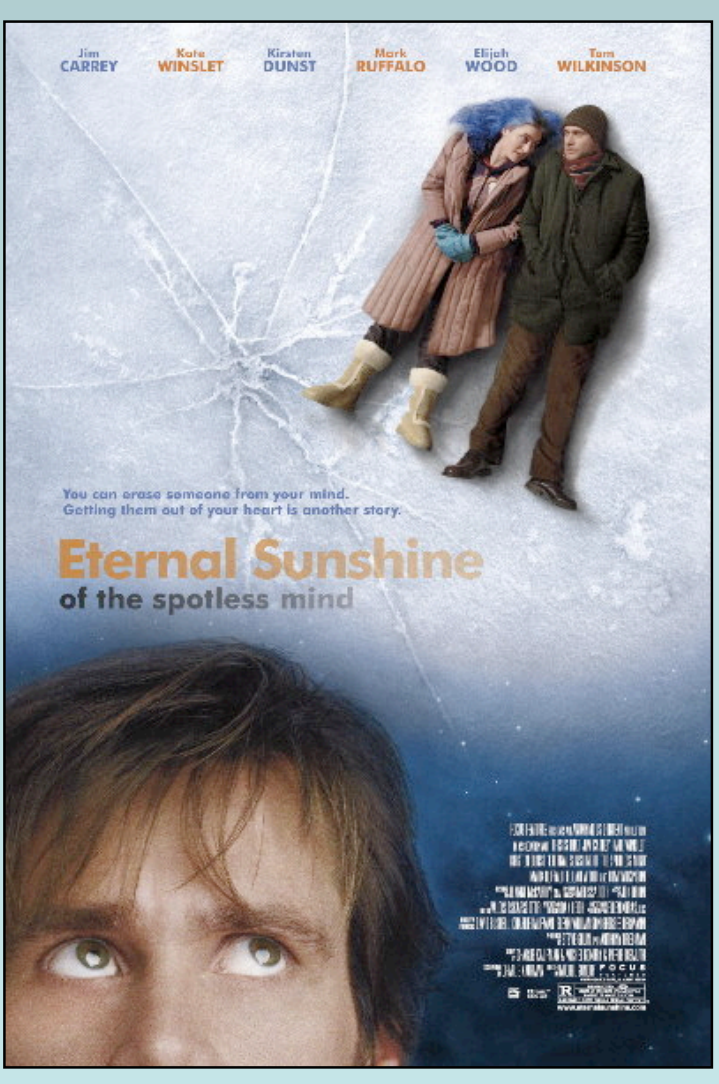

Memory

Uploading 


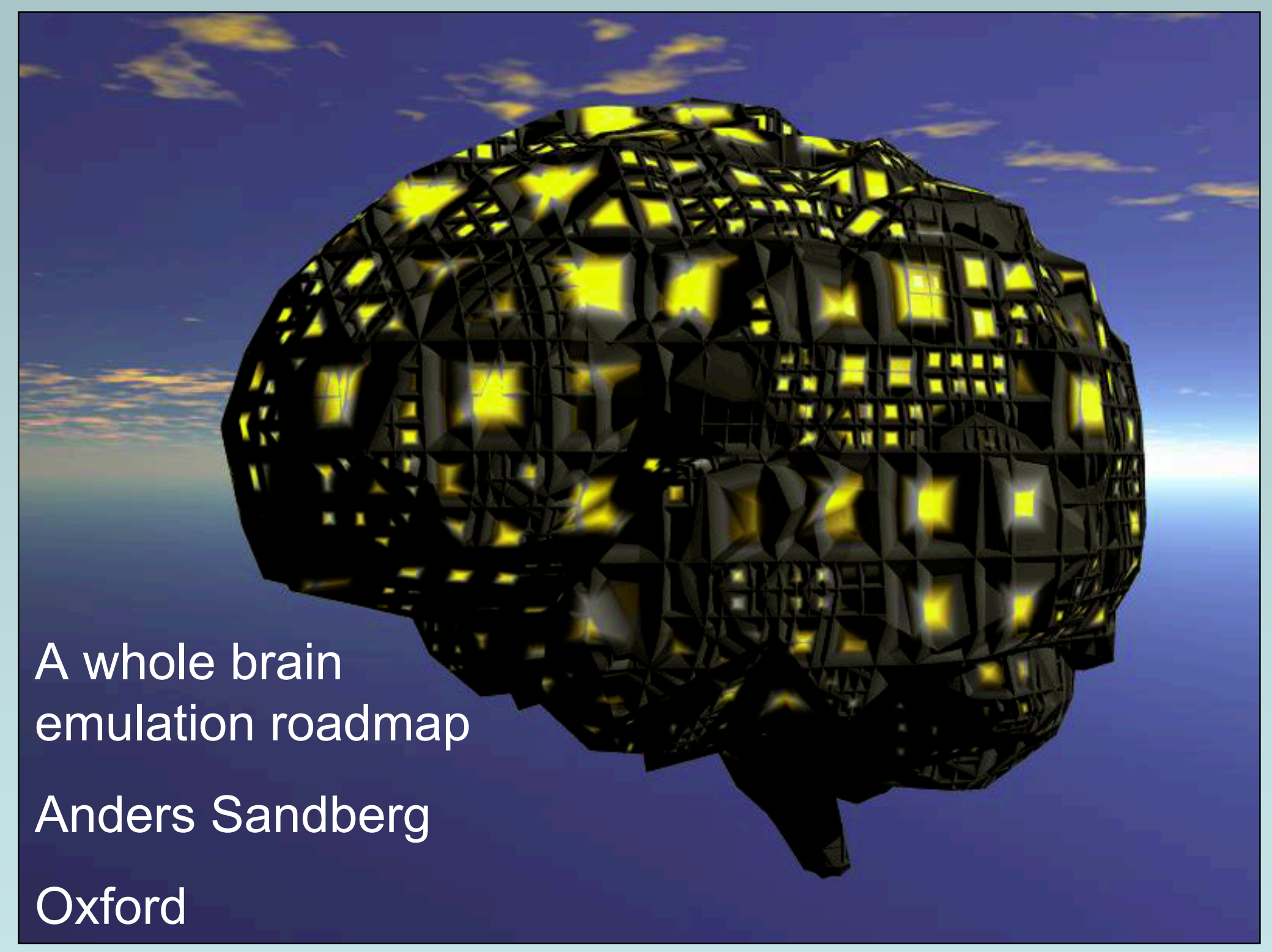

Definitions Cogito... Implants Drugs 


\section{A totally new you}

- Upload

- Scan

- conformation of billions of neurons

- conformation of 1000 s of dendrites on each neuron

- dynamic presence of neurotransmitters at multibillion synapses

- Reconstruct into massive database / program

- Emulate the brain

- Emulate behaviour

- Emulate an individual

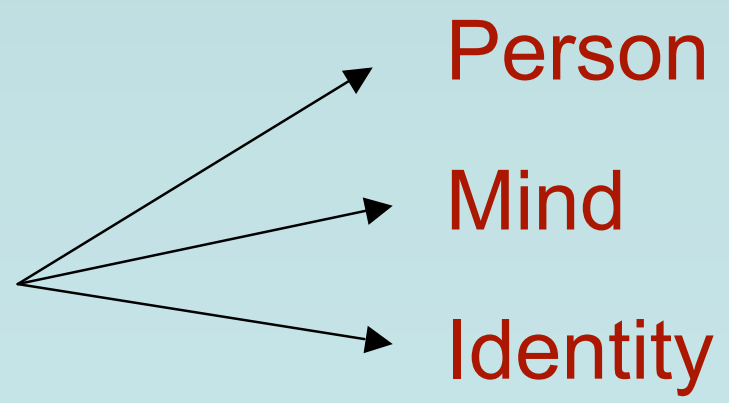

\begin{tabular}{|c|c|c|c|c|}
\hline Definitions & Cogito. & Implants & Drugs & Memory \\
\hline
\end{tabular}




\begin{tabular}{|c|c|c|c|}
\hline Timescale & \multicolumn{2}{|c|}{ Memory } & Power \\
\hline $\begin{array}{l}\text { population } \\
\text { network }\end{array}$ & $50 \mathrm{~Tb}$ & Present & 2032 \\
\hline electrophysiology & $8000 \mathrm{~Tb}$ & 2019 & 2078 \\
\hline $\begin{array}{l}\text { metabolic } \\
\text { information }\end{array}$ & $1,000,000 \mathrm{~Tb}$ & 2029 & 2098 \\
\hline protein information & $10 * 8 \mathrm{~Tb}$ & 2043 & 2112 \\
\hline $\begin{array}{l}\text { molecular } \\
\text { interactions }\end{array}$ & $10 * 14 \mathrm{~Tb}$ & 2069 & 2218 \\
\hline $\begin{array}{l}\text { quantum } \\
\text { behaviour }\end{array}$ & ????????Tb & ???? & ???? \\
\hline
\end{tabular}

Definitions Cogito... Implants Drugs Memory Uploading




\section{Uploading issues?}

- Back ups = Digital immortality

- Beware of viruses

- Legislation against

hacking - anti-autonomy

- Ground Hogg Day possibilities

- Ultimate 'nothingbuttery'

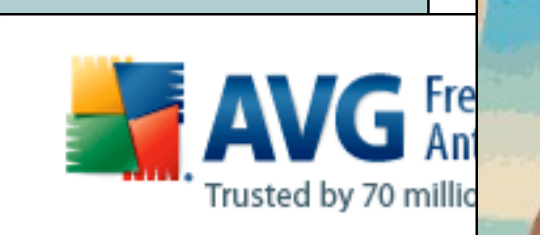

Day
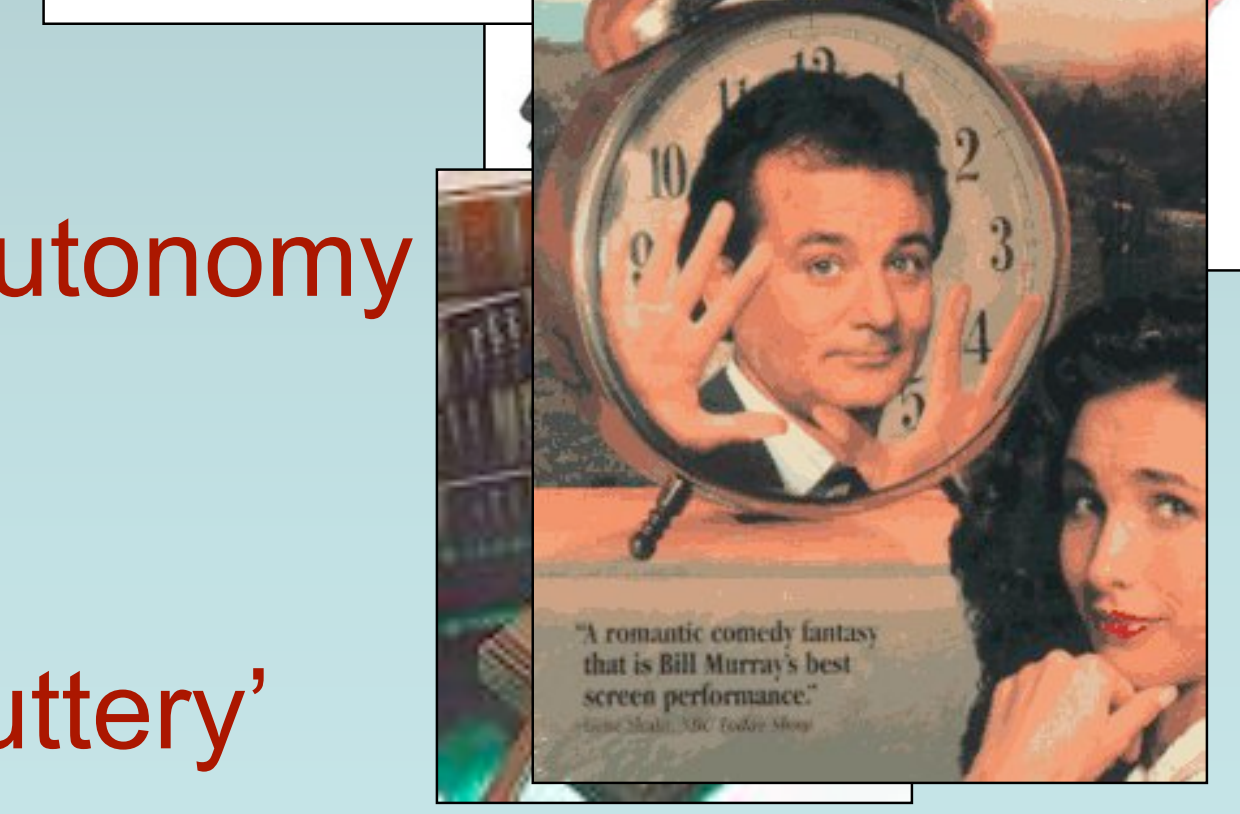

Definitions Cogito... Implants Drugs Memory Uploading 


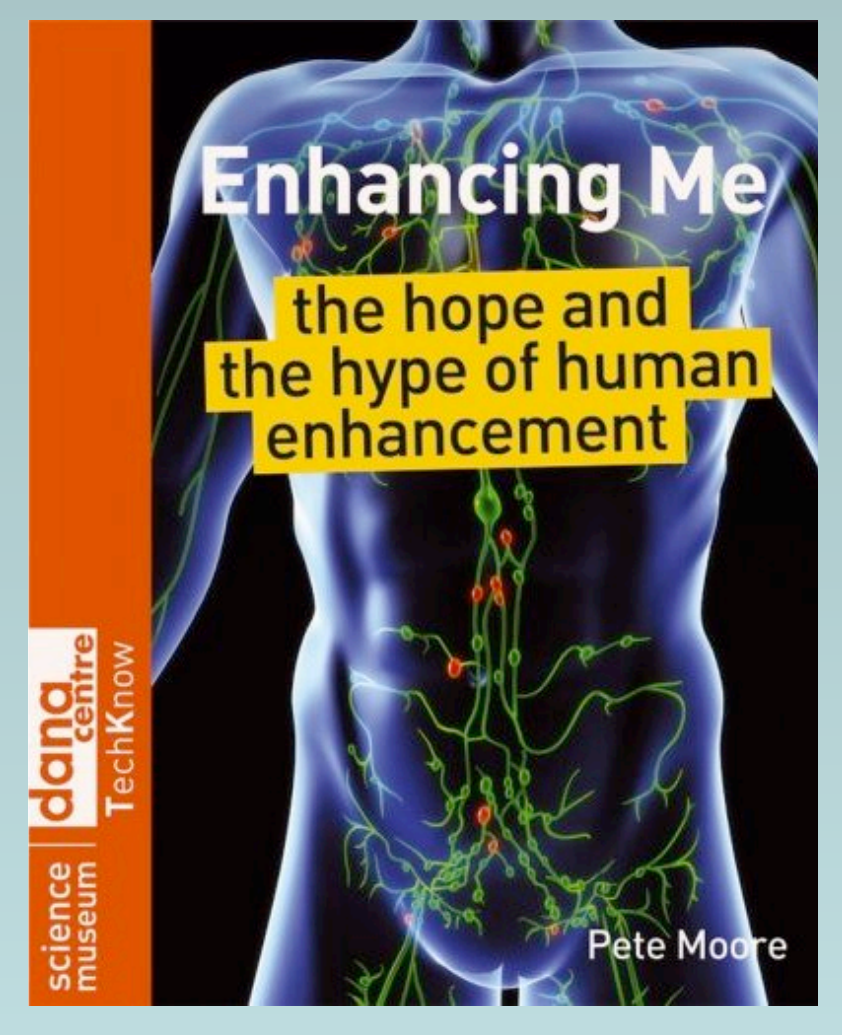

\section{Brain enhancement?}

\begin{tabular}{c|c} 
Personal & Species \\
Hope & Hype \\
Hope & Hype \\
Hope & Hype \\
??? & Hype
\end{tabular}

\begin{tabular}{|l|l|l|} 
Definitions & Cogito... & Implants \\
\hline
\end{tabular}

Drugs

Memory

Uploading 


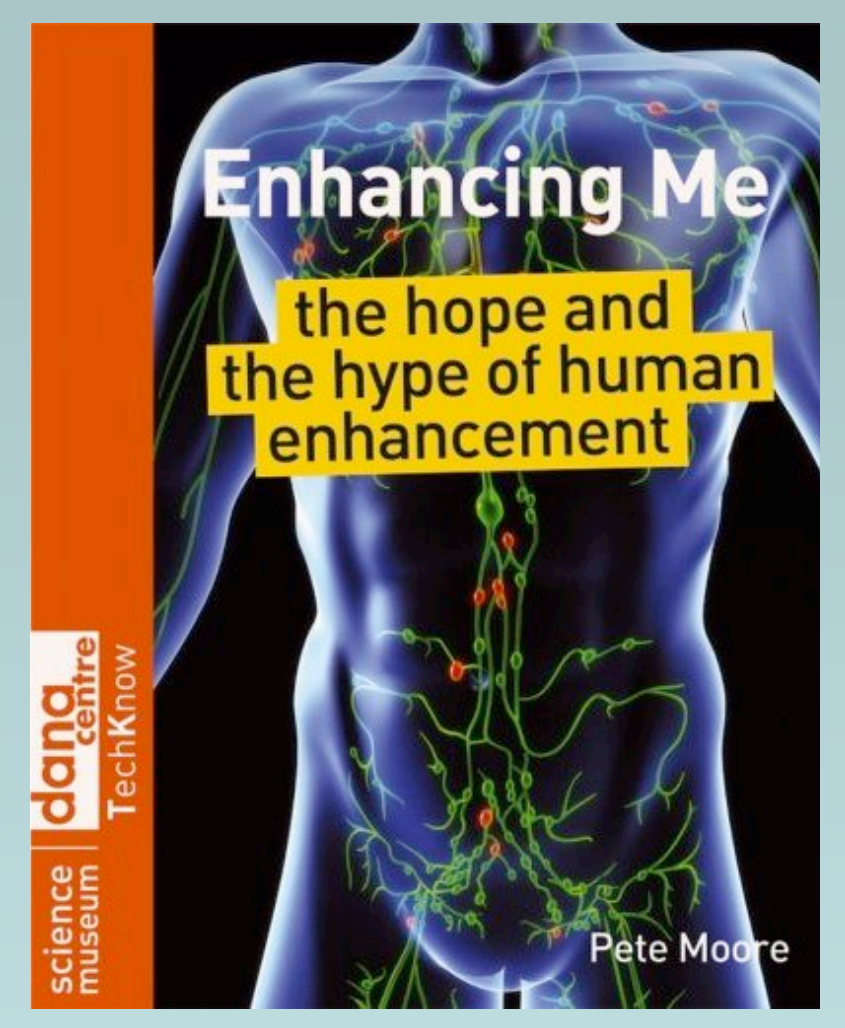

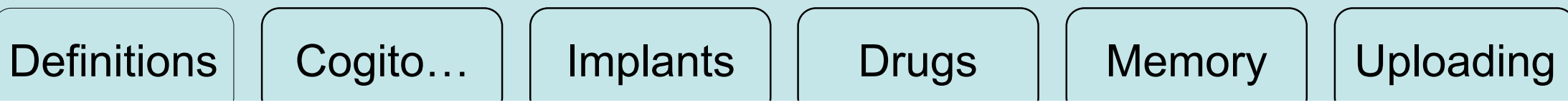




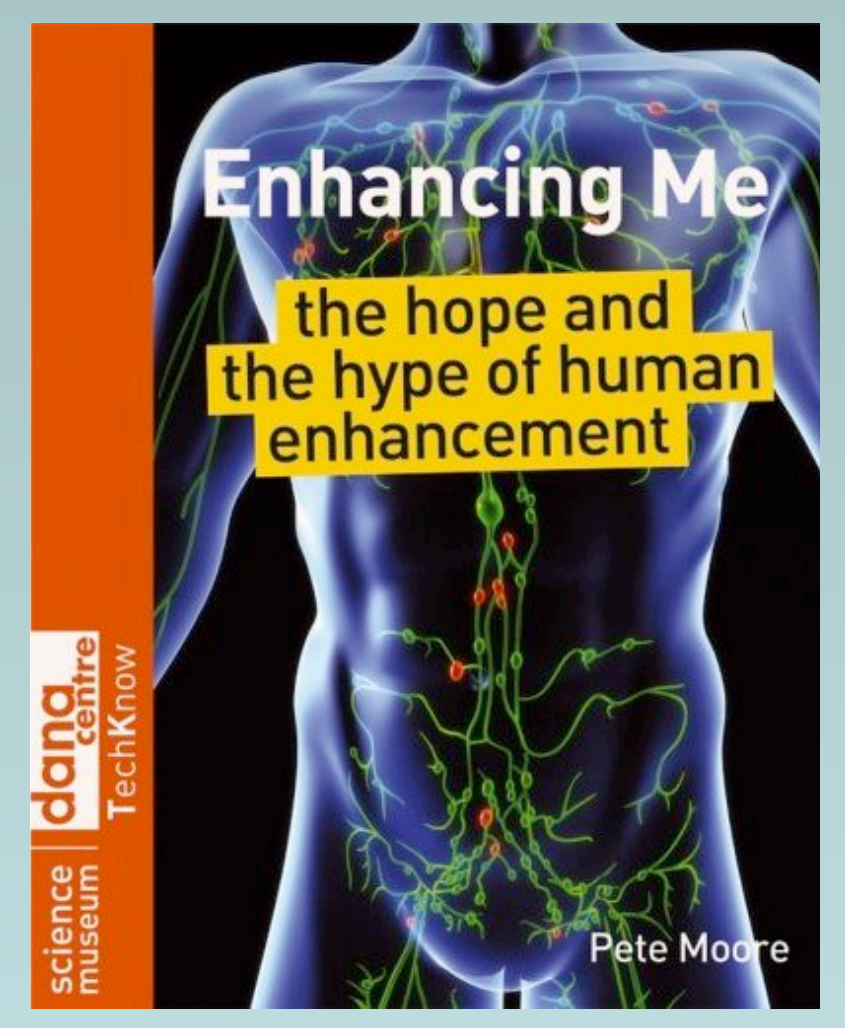

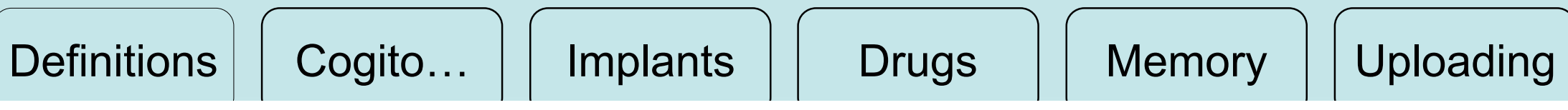

